\title{
Die Atrophie der Follikel und ein seltsames Verhalten der Eizelle.
}

\author{
Von
}

J. Janošik,

Professor an der k. k. böhm. Universitit in Prag.

Hierzu Tafel IX.

Vor einigen Jahren habe ich in Kürze meine Befunde publicirt $\left.^{1}\right)$ iiber das Verhalten der Eizelle in atrophirenden Follikeln bei jungen Säugethieren. Es betrafen meine Untersuchungen besonders Mcerschweinchen. Aus Anlass des Referates von S o b ot ta in Virchow's Jahresbericht fuir das Jahr 1895 S. 97 sehe ich mich veranlasst jene meine Befunde, um nicht missverstanden zu werden, an dieser Stelle zu besprechen und auch noch mancher später gemachten Beobachtungen Erwähnung zu thun.

Nach Flemming befasste sich mit der Atrophie der Ovarialfollikel beim Menschen und verschiedenen Säugethieren S chottlä nder ${ }^{2}$ ). Nach den Angaben beider genannten Autoren kommt die Chromatolysis nur bei jüngeren Follikeln vor, nicht mehr aber bei jenen Follikeln, welche der Reife nalie sind, bei denen schon um das Eichen herum die Corona gebildet wurde. Flem $\mathrm{ming}{ }^{3}$ ) giebt ferner an, dass er diesen Process bei erwachsenen Kaninchen beobachten konnte und meint, dass derselbe nur bei erwachsenen Thieren vorkäme. Nach meinen Untersuchungen kann ich angeben, dass die Chromatolysis in den Granulosazellen am häufigsten bei unreifen Follikeln vorkommt, dass man aber auch bei reifen Follikeln eine solche nachweisen

1) Janošik: Atrofie folikulỉ.etc. Berichte der böhm. Akademie, Prag. 1893, mit französischem Résumė. Kurzes Referat in Bibliographie anatomique. Vol. I. Pag. 90, 1893.

2) Schottländer: Beitrag zur Kenntniss der Follikelatresie etc. Arch. f. mikr. Anat. Vol. 37, 1891 und Vol. 41, 1893.

3) Flemming: Ueber die Bildung von Richtungsfig. etc. Arch. f. Anat. u. Physiol. Anat. Abth. 1885.

Arehiv f. mikrosk. Anat. Bd. 48 
kann. Dieser Vorgang ist normal ebenso verbreitet bei jungen wie bei erwachsenen Thieren, ja er kommt bei jüngeren Thieren noch desshalb in grösserem Maasse vor, weil bei diesen eine viel grössere Zahl von Follikeln untergeht. Bei nähcrer Betrachtung dieses Vorganges kann man sich vielfach von den Ingaben über den Gang der Follikelatresie genannter Autoren überzeugen, dass sie in der That der Wirklichkeit entsprechen, man kann aber auch noch finden, dass viele der Follikelepithelzellen in Zellen sich umwandeln, welche ganz das Aussehen von Leucocyten haben, oder dass die Follikelepithelzellen Zellen solchen Charakters wie die Leucocyten produciren. Hiermit ergïnze ich meine in dieser Bezielung früher gemachte Angabe ${ }^{1}$ ). Dass die Epithelien in einem solchen genetischen Konnexe zu den Lencoeyten stehen, kann man vielfach auch an anderen Stellen sehen. Ich erimnere nur an die Bildung des Thymus, an die Vorgainge in den Tonsillen und den Zungenbalgdrüsen, in verschiedenen Drüsen (Pankreas Lew a s c hew) u. a. Schot t li nder selbst bemerkt: $n$. . sind dem untergehenden Epithel Zellen anderer Herkunft und zwar Wanderzellen beigemischt ..."

Die Angabe, dass die Granulosazellen bei der Follikelatrcsic

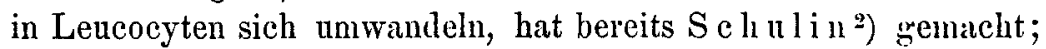
man muss hier aber darauf aufmerksam machen, dass S c h ul in die Granulosazellen von den Stromazellen des Orariums oder aus derselben Quelle wie diese ableitet.

Fragt man, zu welcher Zeit die meisten Follikel untergehen, so kann im Allgemeinen angegeben werden, dass es die Zeit der Trächtigkeit bei den Thieren ist, zu welcher die meisten Follikel normaler Weise nntergehen. Es gelangen zum Anfange der Brunstzeit bei jedem Thiere sehr viele Follikel zur Entwicklung und man kann alle Phasen dieser Entwicklung in einem und demselben Ovarium antreffen. Mit dem Eintritt der Trächtigkeit tritt auch die Involutionsperiode für jene Follikel auf, welche nicht geborsten im Ovarium zurückgeblieben sind. Bemerkt muss ferner noch werden, dass bei jüngeren Thieren zt

1) Janošik: Zur Histologie der Ovarium. Sitzungsberichte der k. Akademie. Wien 1887.

2) Schulin: Zur Morphol. des Ovariums. Arch. f. mikr. Anat. Vol. 19. 1881. 
Die Atrophie der Follikel und ein seltsames Verhalten der Eizelle. 171

dieser Periode melı Follikel gebildet werden und somit auch später mehr derselben in einem und denselben Ovarium untergelıen müssen.

Gerade in dieser Epoche habe ich in den Ovarien junger Thiere gefunden, dass manche Eizellen bevor sie atrophiren noch Theilungen eingehen, und zwar nicht nur in ihren Kernen, sondern auch Theilungen des Zellkörpers erkennen lassen. Neben diesen Theilungen kommen vielfach auch Fragmentirungen des Zellleibes vor, auf welchen Vorgang schon von Pfl i $\mathrm{g}$ e r her verschiedene Autoren bei verschiedenen Wirbelthieren aufmerksam gemacht haben. So finde ich bei S e hulin (l. e.) in cler Fig. 7 ab getheilte Eichen mit Plasmafiagmenten, von denen S c h u lin sagt: "Im Ovarium einer halbwüchsigen weissen Ratte fand ich durch Zerzupfen zwei Eier mit mehrfach getheiltem Dotter und noch erhaltenem und getheiltem Keimblïschen." - In den grössseren Furchungskugeln fanden sich fast stets mehrere Keimblaischen von sehr verschiedener Grösse." Er erinnert an die von Hensen gemachte Bcobachtung iiber die Veriunderungen der Eizelle nach lïngerem Aufenthalte im Uterus. Hensen fand bei einem Kaninchen in abgetrennten Uterushorn an 100 Eichen, deren Protoplasma, wenigstens bei einigen, in 2-8 Segmente gretheilt war. Interessant ist dic Notiz von Grus $\mathrm{d} \mathrm{ew}^{1}$ ) zu lieser Angabe. Neuestens bespricht $\mathrm{H}$ ennegu $\mathrm{y}^{2}$ ) dic Veränderungen an der Eizelle in manchen untergehenden Follikeln, behandelt weiter die Fragmentirung der Eizelle bei verschiedenen Säugethieren, und giebt an, dass die Theilstïcke der Eizelle entweder mit einem Kern angetroffen werden oder ohne denselben. Er konnte keine Eichen auffinden in den ersten Stadien der Theilung, obwohl er Anklänge an dieselbe vorgefunden hat, da die Figuren 11, 18 u. 23 seiner Abhandlung ihm dafür zu sprechen scheinen. Hennegu deutet dann den ganzen Vorgang mit folgendem Satze: On pent considérer la fragmentation de l'ovule .... comme un commencement de développement parthénogénésique", obwohl er früher angeführt hat: "La fragmentation des ovules des Mammifères en voie de régression ne peut être assimilée

1) Grusdew: Versuche über die künstl. Befruchtung von Kanincheneiern. Arch. f. Anat. u. Physiol. Anat. Abth. 1896.

2) Henneguy: Recherches sur l'Atrésie des Follicules etc. Journ. de l'Anat. et de la Physiol. Vol. 30. 1894. 
à la segmentation veritable qui s'observe après la fécondation". In den späteren Stadien der Theilung des Eichens ist der Vorgang immer von einer ganz anderen Art, als die normale Segmentation. Aus dem Gesagten geht hervor, dass Henneguy weit davon entfernt ist, an eine Parthenogenesis in dem Sinne zu denken, wie man aus dem Referate S obotta's entnehmen könnte. Auch ich spreche in keiner Stelle meiner Abhandlung sowohl, wie in dem beigefüigten Résumé von einer parthenogenetischen Entwicklung unbefrucliteter Eier bei den Süugethieren.

In letzter Zeit befasste sich mit der Forschung über parthenogenetische Furchung des Hulhnereies Barfurth ${ }^{1}$ ). Dieser Autor kommt unter Anderem zn dem Schlusse, dass die Segmentirung des Plasmas bei unbefruchteten Hühnereiern nicht als parthenogenetische Furchung aufzufiassen sei, da ihre Produkte keine Kerne besitzen und keine echten Zellen sind. Der ganze Vorgang sei nach Barfurth als lediglich durch physikalisch-chemische Kräfte herbeigeführt zu betrachten. Da nun dieser Autor an Hühnereiern und besonders an virginalen Hühnereiern gearbeitet hatte, so kann man für dieses Object seinen Folgerungen beistimmen.

Neuestens kommt nun Grusdew (l. c.) auch zu dem Schlusse : „Man kann heutzutage kaum zweifeln, dass eine parthenogenetisehe Furchung der Eier bei Süugethieren nie stattfindet. Sie ist wenigstens bisher von keinem Forscher beobachtet worden."

Was nun meine Beobachtungen anbelangt, so will ich in Folgendem die Beschreibung einiger Befunde an Eichen in Ovarien verschiedener Säugethiere geben.

Dass man in Eichen in Follikeln, welche auf verschiedener Höhe der Entwicklung stehen, mehrere Kerne vorfindet, ist von verschiedenen Autoren beobachtet und abgebildet worden. Wenn man solche Eichen mit zwei Kernen in jungen Follikeln auffindet, so kann man immer noch auf die Nähe jener Periode schliessen, in welcher die Epithelzellen, die zu Eizellen werden, sich vielfach durch Theilung vermehrten, und man könnte noch diese Theilung des Kernes der Eizelle, welcher dann die Theilung des Eiplasmas nicht mehr folgt, vielleicht in dem Sinne auffassen,

1) Barfurth: Versuche über die parth. Furchung des Hühnereies. Arch. f. Entwicklungsmechanil. Vol. II. 1896. 
dass der Kern noch genug Lebensfähigkeit bewahrt hat, so dass er noch die eine oder die andere Phase der Theilung sozusagen als Fortsetzung des friiher bestehenden Zustandes, nämlich der Zelltheilung, durchlaufen konnte. Diese Deutung ist schon schwerer, aber noch immerhin zulässig bei jenen Eichen mit zwoi Kernen in weit entwickelten Follikeln. Man kann aber aus solchen Befunden zumindest den Schluss ziehen, das der Eikern a ch in weit oder ganz entwickelten Follikeln die Fähigkeit besitzt sich zu theilen.

Die Vermehrung der Kerne in der Eizelle findet man aber viel häufiger in jüngeren als in etwas älteren oder weit entwickelten Follikeln. In diesen findet man häufiger, dass der Eikern in eine Spindel verwandelt ist, an welcher man die Anorduung der chromatischen sowie der achromatischen Fäden vorfinden kann. Die ganze Kernspindel liegt in diesen Fällen fast immer dicht an der Peripherie des Eichens, senkrecht zur Oberfläche. Auf diesen Umstand hat bereits F lem ming aufmerksam gemacht. Es bleibt bei vielen Eichen bei diesem Vorkommniss; sie zerfallen früher, als dass sie zur weiteren Stufe ihrer Lebensäusserung gelangen könnten. Man findet aber auch Eichen, welche einen höheren Grad der Entwicklung erreicht haben, wie man in der beigefügten Fig. 1 Taf. IX sehen kann. Diese Figur zeigt uns ein Ei mit einem bereits gebildeten kleineren Segmente, welchem man, wenn man es in dieser Stellung und Lage in der Tuba anffinden würde, sicher den Namen und die Bedeutung eines Richtungskörperchens beilegen möchte. Der Körper dieses Gebildes hat absolut dasselbe Aussehen wie das Plasma der Eizelle, ev enthält. chromatische Kernsubstanz, welche bei nüherer Betrachtung in Form von ziemlich dicken kurzen Schleifen angeordnet sich zeigt. Um dieses Gebilde herum kann man auch einen schmalen lichten Hof nachweisen. Von ganz demselben Aussehen ist auch die zurückgebliebene Kernsubstanz und es entspricht das Ganze eigentlich im Wesentlichen jenen Bildern, welche verschiedene Autoren bei normal reifenden Eichen beschreiben oder auch bei bereits befruchteten (vergl. z. B. die Fig. 12 von Sobott ${ }^{1}$ ), wenn man sich nur den Spermakern wegdenkt).

1) Sobotta: Die Befruchtung und Furchung etc. Arch. f. mikr. Anat. Vol. 45. 1895. 
Die Kerne der Granulosizellen des Follikels, in welehen sich dieses Eichen befand, waren an verschiedenen Stellen von dem Zerfall der chromatischen Substanz ergriffen. Der Follikel selbst war soweit entwickelt, dass man denselben für reif erklären könnte. Die Granulosazellen um das Eichen zeigten deutlich eine radiäre Anordnung, die sogenannte Corona.

Das Ovarium, aus welchem dieses Präparat gemacht ist, stammt von einem träichtigen, jungen Kaninchen, bei welchem die im Uterus vorgefundenen Embryonen nahe der Austragezeit waren; sie hatten eine Länge von $9,5 \mathrm{~cm}$.

In der Fig. 2 Taf. IX finclet sich ein Eichen aus cinem ziemlich grossen Follikel aus dem Ovarium eines trächtigen, jungen Heerschweinchens. Der Follikel ist nur etwas kleiner als man reife Follikel bei diesen Thieren vorfindet, die Zellen des Cumulns zeigen aber doch eine stellenweise radiüre Anordnung. Die Granulosazellen sind an jenem dem Cumulus entgegen resetzten Pole von der Chromatolysis ergriffen und man findet zwischen ihnen leucocytenartige Zellen. Die Eizelle selbst besitzt noch eine schwache Membrana pellucida. Da aber diese Nembran in frischen, lebendigen Zustande weich ist und die zugcschricbene derbe Beschaffenheit erst in Folge der einwirkenden Reagentien erlangt, so ist leicht denkbar, dass sie anch bei diesen Eichen im frischen Zustande einen breiteren Hof bildete, als es am konservirten Präparate zu sehen ist. Weil man aber bei Eichen, welche einen noch höheren Grad der Segmentirung zeigen, diese Membran immer schwinden sieht, dagegen aber dieselbe immer derber vorfindet in jenen Fällen, wo das Eichen in toto sozusagen zusammenschrumpft, oder wo dasselbe eine Art Fragmentirung eingeht, so glaube ich berechtigt zu sein annehmen zu können, dass dieser Sehw und der Membrana pellucida mit wirklichen Theilungen des Eichens in irgend einem physiologischen Konnexe steht. Atuch bei dem normaler Weise befruchteten und sich entwickelndem Eichen in der Tuba schwindet diese Membran wohl erst in spüteren Stadien, als welche hier in Betracht kommen. Sobott a findet bei der Maus bei 8-16 Segmenten keine „Zona" mehr. Die Eizelle selbst hat in diesem meinem Falle offenbar eine Theilung durchgemacht, ans welcher zwei Segmente resultiren. Das kleinere Segment ist zwar ziemlich gross im Vergleich zu dem zweiten Segmente, der 
Die Atrophie der Follikel und ein seltsames Verhalten der Eizelle. 175

eigentlichen Eizelle; wiirde man aber ein so verindertes Eichen in der Tuba auffinden, so würde man sicher dazu geneigt sein, dieses Segment für ein Richtungskörperchen anzusehen, unsomehr, als man in dem grösseren Segmente eine schön ausgebildete Kernspindel vorfindet, als würde. sich die Eizelle zur Bilaung: eines zweiten Richtungskörperchen vorbereiten. Die Kernsubstanz in dem kleinen Segmente zeigt ein ähnliches Aussehen, wie jene des Richtungskörperchens in der erwähnten Figur 12 der Abhandlung von Sobotta. Es ist anch anderweitig vielfach bekannt, dass die Kernsubstanz der Richtungskörperchen in verschiedener Weise sozusagen zerfliesst und sie kann desshalb bei vorgeschritteneren Stadien der normalen Furchung gar nicht mehr nachgewiesen werden.

An dieses Stadium reiht sich ein Eichen, welches ich aus einem weit über die Oberfläche des Ovariums reichenden Follikel (ebenfalls von einem träichtigen Meerschweinchen) durch einen Schnitt bei lebenswarmem, soeben dem Thiere entnommenem Organe erhielt. Dieses Eichen besass eine schön entwickelte Corona und wurde am erwärmten Objecttisch im Liquor folliculi selbst untersucht. Das von einer Membrana pellucida umschlossene Eichen war bereits in mehrere Thcilstücke zerlegt; ich sage nuerlegt", weil ich nicht angeben kann, ob in den einzelnen Theilstïcken Kerne vorhanden waren oder nicht, ob es sich also um eine Segmentirung oder eine Fragmentirung handelte. Ich konnte ein grösseres und einige kleinere Theilstiicke hier sehen, welche stark granulirt waren und in denen ich zwar keinen Kern, aber etwas in der Art von Vacuolen nachweisen konnte. Kurz: das Eichen hatte ein Aussehen, welches mich ganz und gar gleich beim ersten Anblick an den Holzschnitt III von Rei ${ }^{1}$ ) erinnerte. Bemerkenswerth ist die bei dieser Gelegenheit von Rein gemachte Angabe, dass in diesem Falle zwar Spermatozoen in der Tube, aber keines in der Nähe des Eichens vorgefunden wurde. Rein spricht bei diesem Eichen vom "Vorhandensein von 4 Körpern, die gar nicht von Richtungskörperchen unterschieden werden konnten", und bemerkt ferner, dass Coste und Bischoff beim Kaninchen 5 Richtungskörperchen nach-

1) Rein: Beiträge zur Kenntniss der Befruchtungserscheinungen etc. Arch. f. mikrosk. Anat. Vol. 22. 1883. 
gewiesen baben. Es ist sicher, dass es sich in diesen Fällen um eine Fragmentirung der Eizelle gehandelt haben muss.

In der Fig. 3 Taf. IX sielit man ein weiteres Stadium der Theilung der Eizelle im Follikel aus demselben Ovariun wie Fig. 2. Der Follikel ist weit in der Atresie vorgeschritten, es sind aber noch ganze Lagen von Granulosazellen zu sehen, welche in verschiedenem Grade der chromatolytischen Veränderung angetroffen werden. Ferner kann man noch in ziemlicher Ausdehnung den Hohlraum für den Liquor folliculi nachweisen, in welchem nunmehr viele der veründerten Granulosazellen lose berumliegen.

Um die Eizelle herum kann keiue Membrana pellucida mehr nachgewiesen werden und es liegt das Eichen frei von einigen Zellen umgeben im Li(unor folliculi. Man sieht, dass dasselbe in zwei gleiche Segmente getheilt ist, von denen, besonders von dem einen, noch Theile in die angrenzenden Schnitte gefallen sind. Jedes dieser Segmente besityt einen runden, wohlbegrenzten Kern, dessen Substanz ein Kernnetz oder Schwammstructur zeigt, mit eingelagerten grösseren Kügelchen von chromatischer Substanz; den Nucleolen. Der Zellleib ist granulirt und erscheint stärker gefürbt im Vergleich mit Eichen aus jüngeren, normal aussehenden Follikeln. Neben diesen zwei grossen Segmenten sind hier noch zwei kleine Segmente vorhanden, von welchen das eine die färbbare Kernsubstanz zu einem etwas unregelmässigen Klümpchen zusammengezogen enthält, wogegen in dem anderen (in der Figur in dem oberen) noch die Form eines etwas zusammengeschrumpften Kernes zu sehen ist. Diese Figur entspricht ganz jener, welehe Asshe ton ${ }^{1}$ ) in seiner Figur 4 wiedergibt und für ein normal sich entwickelndes Eichen nach regelrechter Befruchtung ansieht. Es fehlt bei meiner Figur nur die Membrana pellucida, welche Ass he to n aber auch schon etwas verdünnter zeichnet, als in jüngeren Stadien.

In der Figur 4 kann man nun ein Eichen sehen, welches neben zwei kleinen Theilstïcken, in denen die chromatische Substanz ganz diffus im Plasma zerstreut zu liegen scheint (man kann wenigstens dieselbe an keiner Stelle mehr angehäuft vorfinden), noch drei Segmente aufweist. In dem abgebildeten Schnitte ist ein grosses Segment zu sehen mit einem deutlichen, scharf be-

1) Assheton: A reinvestigation into the early stages of the development of the rabbit. Quarterly Journ. of microsc. sc. 1894. Vol. 37. 
grenzten Kern, in welchem man einen Nucleolus nachweisen kanu, welcher sich intensiv gefürbt hat. Dann ist hier eines der beiden kleineren Segmente zu sehen und ein Theil des zweiten kleineren Segmentes, der andere Theil dieses Segmentes befindet sich im näichsten Schnitte, welcher in der Figur 5 wiedergegeben ist. In dieser Figur sieht man nun den Kern des in Figur 4 nur zum Theil getroffenen Segmentes. Die Kerne der beiden kleineren Segmente erscheinen scharf begrenzt, färben sich aber nur ganz schwach. Eine Membrana pellucida ist nicht mehr vorhanden. Wenn man ein solches Eichen in der Tube auffinden möchte, so wïrde man von einer inaequalen Furchung sprechen, bei welcher aus den beiden ursprünglichen Segmenten der Figur 3 das eine sich bereits weiter getheilt hat, das andere aber noch nicht. Neben diesen drei Segmenten mit Kernen sind hier noch die zwei kleinen Theilstïcke, welche man für Richtungskörperchen ansehen könnte. Solche Befunde sind vielfach an befruchteten, normal sich entwickelnden Eichen beschrieben worden. Der Follikel, in welchem dieses Ei sich betindet, ist fast ganz atresisch, es bleibt nur ein klciner Hohlraum um das Eichen herum uibrig.

Neben diesen Stufen der Theilnng habe ich auch noch weitere auffinden können. In der Figur 6 ist cin Eichen abgebilclet, welches in viele untereinander fast gan\% gleiche Segmente getheilt ist. Dass man hier von einer Theilnng und nicht nur von einer Fragmentirung sprechen kamn, das rechtfertigt mir der Umstind, dass ich in den Segmenten Kerne vorfinde. Dieselben sind scharf begrenzt, fürben sich aber schwach. Zwischen den einzelnen Segmenten ist ein kleiner, spaltförmiger Hohlraum wie eine Art von einer Furchungshöhle. Diesen Spalt ausgenommen entspräiche diese Figur jener von Sobotta (Taf. VI Fig. 30). Die Segmente auf der linken Seite der Figur sind zum Theil in den folgenden Schnitt gefallen und anch ihre Kerne. Neben solchen Eichen mit fast untereinander ganz gleichen Segmenten finde ich auch solche mit ungleichen, wie aus der Figur 7 zu sehen ist. Das Eichen, von dem diese Figur stammt, ähnelt ganz der Figur 29 der Abhandlung von Sobotta. Beide in Figur 6 und 7 abgebildete Eichen liegen in Follikeln, welche noch einen ziemlich grossen mit Liquor gefüllten Hohlraum zeigen. Neben diesen hier ausführlicher besprochenen Befunden, 
könnte ich noch aus verschiedenen Ovarien eine ganze Reihe auf verschiedener Höhe der Theilung stehenden Eichen auffülren, neben welchen auch wahre Fragmentirungen angetroffen werden. Nitunter trifft man auch auf Eizellen, welche in mehrere ungleiche Theile zertheilt sind, in denen man in einigen Kerne nachweisen kann, in anderen nicht. Die kernhaltigen sind zumeist die grösseren Theilstïcke. Bemerkenswerth ist, dass in jenen Ovarien, in denen ich wirkliche Theilung'en nachweisen konnte, jene Atrophie der Eizelle, bei welchen die Membrana pellucida erhalten bleibt und von welcher verschiedene Antoren berichten und ich selbst seiner Zeit') ebenfalls meine Beobachtungen veroffentlicht habe, fast gar nicht angetroffen wird.

Wie nun diese Theilungen der Eizelle im Ovarium vor sich gehen, kanm ich nicht näher angeben, muss hier aber bemerken, dass ich in meinen diesbeziiglichen Präparaten bei der weiteren Theilung keine Kernspindeln vorfinde. $E_{s}$ ist das um so auffälliger, als ich in den Anfangsstadien sehr viel Eichen mit Kernspindeln sehen kann.

Was nun die Ursache anbelangt, warum die oben beschriebenen Theilungen gerade bei jungen Thieren angetroffen werden, so habe ich die Vermuthung ansgesprochen, dass das vielleicht mit jenem Befunde zusammenhüngt, den ich fast bei allen Eichen in diesen Ovarien die Gelegenheit hatte zn machen, nämlich dass in allen ein ziemlich deutlicher Nebenkern nachgewiesen werden kann. In der Figur 8, welche ich meiner oben angeführten Arbeit im Lichtdruck beigegreben habe, kann man einen jungen Follikel sehen, in welchem im Eichen neben dem Hauptkerne ein deutlicher Nebenkern zu sehen ist. H e n n eguy, welcber auch diesen Punkt berïhrt, meint, dass in dem Nebenkerne hier die Ursache nicht liegen kann, da derselbe wohl bei jungen Eichen angetroffen wird, bei Eichen aber in weit entwickelten Follikeln nicht mehr nachzuweisen ist. Ich habe nun diese Ovarien, von denen ich Serienschnitte besitze, anch in dieser Hinsicht durchgesucht und finde, dass hier auch in ganz entwickelten Follikeln in der Eizelle dieses Gebilde, welches ich als den Nebenkern ansehe, angetroffen wird. In der Figur 8 ist ein Eichen gezeichnet, bei welchem die Stellung der dem Eichen an-

1) l. c. sub 4 . 
Die Atrophie der Follikel und ein seltsames Verhalten der Eizelle. 179

liegenden Granulosazellen schon selbst beweist, dass dasselbe einem weit entwickelten Follikel entnommen sein muss und man findet in diesem Eichen dennoch einen Nebenkern, welcher dicht dem Kerne anliegt. Ich bemerke nur, dass dieses keine Ausnahme ist, da ich dieses Gebilde fast in allen Eichen nachweisen kann. Es kommt vor, dass es nicht in demselben Schnitte liegt wie der Hauptkern, dann kann ich es aber in den angrenzenden Schnitten finden.

Anklänge an diese eben besprochenen Theilungen habe ich, zwar nicht in einer so kompleten Serie, vorläufig noch bei der Katze und Fledermans vorgefunden; Mäuseovarien labe ich von jungen Thieren noch nicht in einer grösseren Anzahl zu untersuchen Gelegrenheit gehabt.

Ich habe ferner bei meinen Objecten gefunden, dass die Eizelle, mag sie sich theilen oder fragmentiren oder schollig zerfallen, immer $z$ Grunde geht, ich will aber nicht behaupten, dass der Ausgang immer ein solcher scin muss. Man könnte vielleicht gelegentlich bei pathologischen Befunden die eben mitgetheilten Beobachtungen mit in Betracht ziehen.

Aus dem eben Vorgeführten folgt:

1. Die Eizellekann im Ovarium durchregelrechten Process die Richtungskörperehen oder denselben gan grleichende Gebilde liefern. Dieses geschieht am häufigsten bei jungen Thieren, kann aber a u ch in Ovarien älterer Thierevorgef unden werden.

2. Nach der Bildung dieser Gebilde kann die Eizelleim Ovarium, also ohnejede Befruchtung, noch weiter sich theilen, wobeidie aus der TheiI ng resultirenden Segmente Kerne enthalten.

3. Dieser Process der Theilung kann bis zur Bildung einer grösseren Anzahl von Segmenten führen, welche alleKernebesitzen und untereinander fast ganz gleich sein können, oder

4. eskann die Theilung aber auch zur Bildung. von Segmenten führen, welche untereinander ungleich gross sind, die aber alle Kernebesitzen und somit den Charakter von Zellen haben.

5. Nebendiesen wirklichen Theilungen kom- 
men auch vielfach nur Fragmentirungender Eizelle vor (welchen Vorgang man besonders bei älteren Thieren vorfindet) oder es kann a u ch die Eizelle ${ }^{\text {schollig zerfallen". }}$

6. Mit der fortschreitenden Theilung der Eizelle gehtauch die Membrana pellucidaverloren, wie es bei der normalen Entwicklung nach der stattgefundenen Befruchtung in der Tuba geschieht. (Sobotta bei der Maus).

Es gehört meiner Meinung nach nicht in das Reich der Unmöglichkeiten, ja es ist eigentlich sehr wahrscheinlich, diss ein Eichen, wenn es in die Tube gelangt und auch nicht befruchtet wurde, dass es dieselben Phasen der anfäinglichen Furchungsstadien durchlaufen kann ebenso, wie wenn es im Ovarium zulrückgehalten worden wäre. Ich behaupte es nicht und habe es nicht behauptet, da ich durch wirkliche Befunde nicht beweisen kann, lass ein solches ausgetretenes Eichen wirklich diese Phasen ohne Befruchtung durchmacht, ich meine nur, dass man beim Studium der ersten Furchungsstadien leicht auch solche Eichen mit bekommen könnte und dieselben dann auch in den normalen Gang der Theilung von befruchteten Eichen einschalten könnte. Ich will auch weiter nicht darauf eingehen, wie man diese Befunde mit den verschiedenen Theorien über die Bedentung der Richtungskörperchen in Einklang bringen sollte, wo nach der Bildung von Richtungskörperchen oder wenigstens diesen gleichenden Gebilden, die Eizelle dennoch die Möglichkeit besitzt sich weiter zu theilen. Es ist leicht, die diesbezïglichen Deduktionen zu machen.

Ich möchte hier nur noch auf die Abhandlung ron $\mathrm{M}_{\text {or }} \mathrm{gan}^{1}$ ), sowie auch auf die zu dieser Arbeit gemachte Bemerkung von $\mathrm{R}$ o $\mathrm{x}$ aufmerksam machen, um darauf hinweisen zu können, von wie grosser Tragweite auch ziemlich geringe Modifikationen der Lebensverhältnisse der Zelle für die morphologischen Befunde sind, die gelegentlich gemacht werden.

Man muss doch, will man der Wahrheit näher treten, anch auf solche nicht gewöhnliche (ich will nicht sagen abnormale,

1) Morgan: The production of artificial astrophaeres. Arch. für Entwicklungsmech. Vol. 3. 1896. 
Die Atrophie der Follikel und ein seltsames Verhalten der Eizelle. 181

lieber abweichende) Befunde sein Augenmerk richten, und dieselben in den Kreis der Beobachtıngen ziehen.

\title{
Erklärung der Abbildungen anf Tafel IX.
}

Alle Figuren sind projicirt mit: Reichert Hartapochromat $4 \mathrm{~m} / \mathrm{m}$. Ocular III., Details sind eingezeichnet mit Reichert Objectiv 8 a.

Fig. 1. Ein Eichen aus einem reifen oder der Reife nahen Follikel aus dem Ovarium eines jungen, trüchtigen Kaninchens. (Liinge der Embryonen $9.5 \mathrm{~cm}$ ).

Fig. 2-8 sind aus einem und demselben Ovarium eines jungen, träichtigen Meerschweinchens.

(Aus dem anatomischen Institut der Jagellonischen Universitit in Krakau.)

\section{Ueber das Verhältniss der Centrosomen zum Protoplasma ${ }^{1}$.}

\author{
Von
}

K. Kostaneeki und M. Siedlecki.

Hierzu Tafel $\mathrm{X}$ und XI.

Die Untersuchungen über die Befruchtung des Eies von Ascaris megalocephala baben eine epochemachende Rolle in der cytologischen Literatur gespielt. Die Arbeiten von van Beneden und Boveri über dieses Objekt haben nicht nur einen mächtigen Umschwung in unseren Vorstellungen von der Befruchtung

1) Vorgetragen in der Sitzung der mathematisch-naturwissenschaftlichen Klasse der Akademie der Wissenschaften in Krakau vom 4. März 1896 sowie während der Versammlung der Anatomischen Gesellschaft in Berlin am 20. April 1896, woselbst die entsprechenden Präparate demonstrirt wurden. 